\title{
60. \\ Evaluación del consumo de energía eléctrica de acuerdo a la arquitectura bioclimático mediante el Modelo ASHRAE y Gauss T- Student
}

\section{Evaluation of electricity consumption according to the bioclimatic architecture using the ASHRAE Model and Gauss T-Student}

\author{
Cristian Cristóbal Cuji Cuji ${ }^{*}$, Henry David Sisa ${ }^{1}$ \\ ${ }^{1}$ Facultad de Ingeniería Eléctrica, Universidad Politécnica Salesiana, Ecuador \\ *Autor de correspondencia: ccuji@ups.edu.ec
}

\begin{abstract}
RESUMEN- La presente investigación evalúa los resultados del consumo de energía eléctrica en ambientes normales con el fin de alcanzar condiciones óptimas de confort, aprovechando las condiciones bioclimáticas y aire acondicionado, proponiendo el modelo Gauss T-Student como método para estimar la frecuencia de trabajo de aire acondicionado, con condiciones de temperatura, humedad relativa y velocidad del viento desde el 8 de agosto del 2018 hasta el 8 de agosto del 2019 de la localidad de GuayaquilEcuador. La idea clave es combinar el conocimiento previo del modelo The American Society of Heating, Refrigerating and AirConditioning Engineers (ASHRAE), como método para el análisis de arquitectura bioclimática por ganancias de energía térmicas, para estimar la temperatura de sensación interna y controlarla por medio del modelo probabilístico. Los resultados del consumo eléctrico son aplicables a simulación del sector comercial para satisfacer las necesidades de confort.
\end{abstract}

Palabras clave-Arquitectura bioclimática, confort, consumo eléctrico, Gauss, T-Student, ingeniería eléctrica, termodinámica.

ABSTRACT - The present investigation evaluates the results of the consumption of electric power in normal environments in order to reach optimal comfort conditions, taking advantage of bioclimatic conditions and air conditioning, proposing the Gauss-TStudent model as a method to estimate the air working frequency conditioning, with conditions of temperature, relative humidity and wind speed from August 8, 2018 to August 8, 2019 in the town of Guayaquil-Ecuador. The key idea is to combine prior knowledge of The American Society of Heating, Refrigerating and Air-Conditioning Engineers (ASHRAE) model, as a method for the analysis of bioclimatic architecture by thermal energy gains, to estimate the temperature of internal sensation and control it by middle of the probabilistic model. The results of electricity consumption are applicable to simulation of the commercial sector to meet the needs of comfort.

Keywords-Bioclimatic architecture, comfort, power consumption, Gauss, T-Student, electrical engineering, thermodynamics.

\section{Introducción}

La presente investigación se estudia el consumo de energía eléctrica de acuerdo con la arquitectura bioclimática de la región, además esta investigación es parte de la unidad de titulación y se presentan resultados a fondo, donde se propone el modelo Gauss T-Student, para sistemas óptimos de confort en función a la arquitectura bioclimática y el uso de acondicionadores de aire dentro del sectores comerciales [1-4]. Los métodos probabilísticos proporcionan información real sobre el comportamiento de un sistema y manejan gran cantidad de datos, como una forma de manejar incertidumbres [5], [6], además se aplica en distribuciones de variables continuas como temperatura y tiempo. Otros autores proponen como métodos para estimar el consumo de energía eléctrica por efecto de datos climáticos como cuadratura de Gauss, regresión lineal, regresión polinomial, probabilidad Kullback-Leibler, calculo integral bajo la curva y mecanismos de predicted Mean Vote para determinar el aumento de la demanda eléctrica para satisfacer las necesidades de confort [7-9]. Para

Citación: C. Cuji y H. Sisa, "Evaluación del consumo de energía eléctrica de acuerdo a la arquitectura bioclimático mediante el Modelo ASHRAE y Gauss TStudent", Revista de I+D Tecnológico, vol. 17, no. 1, pp. (no_modificar), 2021.

Tipo de artículo: Original. Recibido: 27 de febrero de 2020. Recibido con correcciones: 17 de junio de 2020. Aceptado: 17 de junio de 2020. DOI.

Copyright: 2021 C. Cuji y H. Sisa. This is an open access article under the CC BY-NC-SA 4.0 license (https://creativecommons.org/licenses/by-nc-sa/4.0/). 
encontrar un sistema óptimo de confort, con la finalidad de satisfacer las necesidades ambientales normales del sector comercial, los sistemas de confort valoran la capacidad de mantener las condiciones ambientales dentro de los edificios, adecuando el ambiente para el confort de los usuarios [10-12]. En la investigación el inconfort representa temperaturas de sensación térmica (TST) iguales o mayores a $26^{\circ} \mathrm{C}$ o menores o iguales a $22^{\circ} \mathrm{C}$; la temperatura de sensación térmica es un incremento de temperatura interna por ganancias térmicas del exterior al interior del edificio [13-17], y se calculara mediante el modelo The American Society of Heating, Refrigerating and Air-Conditioning Engineers, que también calcula la capacidad de aire acondicionado se requiera para eliminar la temperatura de sensación térmica interna [18], [19]. El uso de aire acondicionado representa el 40\% consumo energético [20], [21], por esto se emplea estos métodos sobre datos estadísticos, como objetivo determinar el efecto del consumo de energía eléctrica en sistemas óptimos de confort en función a la arquitectura bioclimática y aire acondicionado dentro del sector comercial, en un entorno simulado. reduciendo las facturas de energía eléctrica [22-24]. El método The American Society of Heating, Refrigerating and Air-Conditioning Engineers, establece la capacidad de aire acondicionado por medio de ganancias térmicas y conservación de la energía, aprovechando las condiciones bioclimáticas del exterior [13], [25-28]. Para este estudio, la compensación con aire acondicionado determina la cantidad y capacidad para obtener confort y evaluar el consumo de energía eléctrica [29-32], [33].
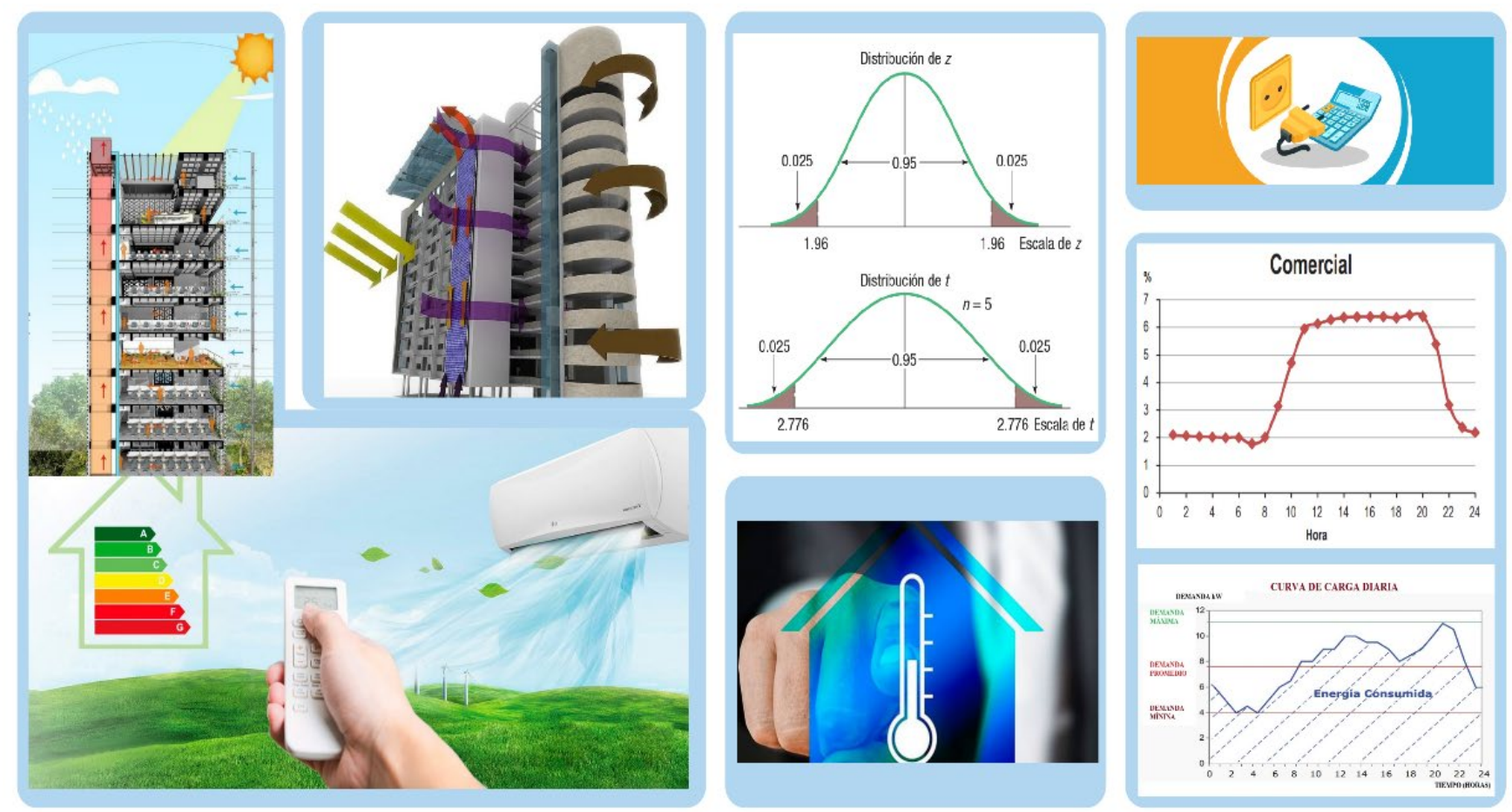

Figura 1. Sistema óptimo de confort bajo arquitectura bioclimática y aire acondicionado.

\section{Modelamiento de estimación de consumo eléctrico del sistema}

El empleo de aire acondicionado por sí solo, genera un consumo de energía eléctrica elevado, para disminuirlo se requiere de las características bioclimáticas, aprovechando todas las condiciones naturales exteriores, la posición sobre el nivel del mar (msnm), latitud y longitud, de ubicación de la edificación [11], [25], [26].

También se considera los materiales de construcción (paredes, ventanas, puertas), materiales de aislamiento y ductos [31], [34-37]. Los datos de temperatura, humedad relativa y velocidad del viento se toman de la base de datos del Instituto Nacional Meteorología e Hidrología del Ecuador (INAMHI). Toda esta información analiza 
en un entorno simulado en software Matlab. Para el análisis se presentan en este documento un escenario, que corresponden a la ciudad y se detalla a continuación:

El escenario se encuentra ubicado en la ciudad de Guayaquil, latitud -2.219213, longitud -79.888479, sector Naval Sur, altitud $4.02 \mathrm{msnm}$.

El análisis del consumo de energía eléctrica del escenario se realiza de la siguiente manera:

- Modelo Matemático ASHRAE

- Gauss -T-Student.

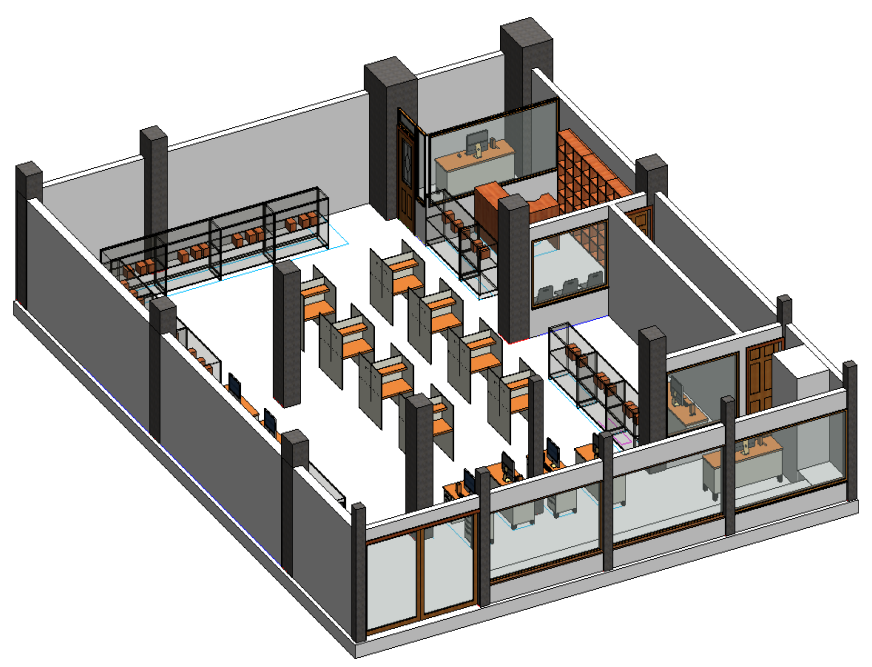

Figura 2. Análisis de Planta, aprovechamiento bioclimático.

\subsection{Modelo Matemático ASHRAE}

A partir de la adquisición de datos de temperatura, humedad relativa y velocidad de viento, de acuerdo con la posición de la localidad se obtienen ganancias térmicas de exterior a interior de edificación. Esto se consigue por medio de análisis termodinámicos de conservación de energía. Es por esto por lo que se propone como método de resolución el modelo ASHRAE, para calcular todos los parámetros que involucran el aprovechamiento de arquitectura bioclimática existentes. La arquitectura bioclimática está ligada a la construcción ecológica y define parámetros de aislamiento naturales para satisfacer la necesidad de confort y reducir el uso de la energía convencional. Los parámetros como, aislamiento de paredes, techos y pisos; cristales como ventanas y lucernarios; ventilación por velocidad del viento natural; pueden definir las ganancias de calor al interior del edificio [11], [25], [26]. La investigación propone el proceso de resolución de la siguiente manera del modelo ASHRAE y se describe en la Tabla 1.:
Se debe conocer la ubicación de localidad del edificio, los datos bioclimáticos del exterior, para proceder a calcular diferentes incógnitas de confort ambiental, como por ejemplo presión atmosférica, humedad de saturación, punto de roció y entalpia. A partir de la variable de entalpia se puede dar a conocer la temperatura interna en condiciones iniciales sin ganancia de calor [11], [25], [26].

Se debe conocer las características de construcción del edificio, espesor y área de las paredes, techos, y pisos; espesor y área de los cristales, espesor y área de ductos, si existiesen [11], [25], [26].

Tabla 1. Algoritmo ASHRAE para determinar el comportamiento bioclimático.

\begin{tabular}{|c|c|}
\hline Paso 1 & $\begin{array}{l}\text { Adquisición de datos: temperatura, humedad } \\
\text { relativa y velocidad de viento. Msnm, latitud } \\
\text { (GMS). }\end{array}$ \\
\hline Paso 2 & 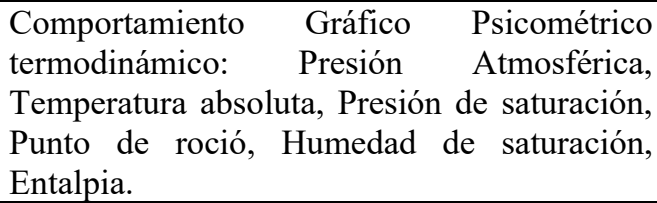 \\
\hline Paso 3 & Ganancias térmicas edificación. \\
\hline Paso 4 & $\begin{array}{l}\text { Ganancias térmicas por conducción a través de } \\
\text { la estructura }(Q S T) \text {. }\end{array}$ \\
\hline Paso 5 & $\begin{array}{l}\text { Ganancias térmicas por conducción interna } \\
(Q I S) \text {. }\end{array}$ \\
\hline Paso 6 & $\begin{array}{l}\text { Ganancia térmica por radiación solar cristales } \\
(Q V) \text {. }\end{array}$ \\
\hline Paso 7 & Ganancia térmica por usuarios $(Q P)$. \\
\hline Paso 8 & Ganancia térmica por iluminación $(Q A)$. \\
\hline Paso 9 & Ganancia térmica por equipos $(Q E q)$. \\
\hline Paso 10 & Ganancia térmica por filtración de aire $(Q I F)$. \\
\hline Paso 11 & $\begin{array}{l}\text { Cálculo Total de ganancias térmicas. } \\
(Q T C A=S T+Q V+Q I S+Q P+Q A+ \\
Q E q+Q I F=B T U / H) .\end{array}$ \\
\hline & $\begin{array}{l}\text { Cantidad de equipos aire acondicionado } \\
\text { capacidad de aire acondicionado }(\text { CapAA). }\end{array}$ \\
\hline
\end{tabular}

Paso 12 Si $Q T C A>\operatorname{CapAA}$

$$
\text { Nequipos }=\frac{Q T A C}{\operatorname{CapAA}}
$$

Fin si

Las ganancias térmicas por condición a través de exterior a interior e interior a interior, se involucran variables como diferencia de temperatura del exteriorinterior, espesor y área de cada uno de los elementos de construcción. Las ganancias térmicas por radiación involucran a todos los elementos de cristal que posea el 
edificio e iluminarias. Las ganancias por convección se obtienen a partir de la transformación de energía por cada elemento como iluminación, aparatos eléctricos u otros y personas. La ganancia térmica por infiltración de aire se obtiene a partir de la velocidad del viento que rodea al edificio, además es muy pequeña y su almacenamiento no existe al interior, pero si se involucra por términos de renovación del viento.

La sumatoria de todas las ganancias térmicas, dan lugar a la obtención de temperatura de sensación térmica, la que define si el interior de una habitación se encuentra en comodidad, si existe excedentes, es necesario la instalación de equipos que acondicionen el confort. Además de conocer las ganancias de calor totales internas, el modelo también es útil para la determinación de la capacidad de aire acondicionado.

\subsection{Modelo Gauss, T-Student}

Las aplicaciones para variables inciertas son base de datos que administran objetos en movimiento donde no es posible determinar las posiciones exactas de los objetivos en cada punto del tiempo. A partir de los datos experimentales de temperatura se predicen los coeficientes de tiempo y con qué frecuencia pueda ocurrir el evento. Se puede suponer una función de distribución de densidad que se puede usar para aproximar el valor de tiempo exacto observado [5], [6], [9], [38]. La función de densidad de probabilidad gaussiano con respecto a un valor medio y una deviación estándar se define con la ecuación (1) para modelar la incertidumbre a partir de la variación de temperatura.

En los sistemas de energía, la confiabilidad juega un papel importante, es por esto que estimar el correcto de tiempo aplicado de los equipos de aire acondicionado, se verá como resultado el aproximado consumo eléctrico bajo la aplicación de estos equipos con respecto al tiempo [39], [40].

$$
F T(T)=\frac{e \frac{-(T-u T)}{2 * S D^{2}}}{S D^{*} \sqrt{2 * P I}}
$$

Dónde:
FT(T) Función de densidad de probabilidad gaussiana temperatura, $T \in R$.

$\mathrm{T} \quad$ Muestras aleatorias Temperatura $\left[{ }^{\circ} \mathrm{C}\right]$.

uT Valor de temperatura esperado $\left[{ }^{\circ} \mathrm{C}\right]$.

SD Desviación estándar de temperaturas.

Dónde:

$$
u T=\frac{\sum_{i=0}^{n} T_{i}}{n}
$$

Está comprobado que la distribución de probabilidad de la temperatura está sujeta a una distribución de densidad normal de acuerdo con la ecuación (1), si cumple:

$$
\sum_{i=0}^{n} F T\left(T_{i}\right)=1
$$

Cuanto sea mayor el periodo de observación mayor será la probabilidad. Sin embargo, la variación de la variable temperatura con respecto al tiempo es propensa a cambios significativos. De acuerdo con un pronóstico del tiempo, por cada hora del día corresponderá a un cambio de temperatura, considerando que ciertas horas del día puedan permanecer constantes. Es por esto que propone analizar mediante la combinación de probabilidad de densidad T-Student para el cálculo del tiempo con el diseño de muestras aleatorias más pequeñas[7], [38].

$$
Z T=\frac{T_{\min }-u T}{S D}
$$

Dónde:

ZT Función gaussiana estandarizada.

$\mathrm{T}_{\text {min }}$ Muestras mínima Temperatura $\left[{ }^{\circ} \mathrm{C}\right]$.

uT Valor de temperatura esperado $\left[{ }^{\circ} \mathrm{C}\right]$.

SD Desviación estándar de temperaturas.

La ecuación (4), describe la función $\mathrm{Z}$ gaussiana estandarizada, con respecto al comportamiento de las muestras de temperatura, los resultados de esta ecuación dan paso al estudio de la probabilidad de fracaso que exista en una distribución. Es decir, la probabilidad de $\mathrm{ZT}$, corresponden a los valores que no son esperados o 
que no se consideran para la investigación. Por otro lado, se conoce:

$$
\begin{gathered}
P(Z T)=P(F) \\
P(E)=1-2 P(F)
\end{gathered}
$$

Dónde:

P(F) Probabilidad de fracaso en ZT.

P(E) Probabilidad de éxito

La ecuación (6), describe la cantidad de muestras están contenidas por la probabilidad de éxito. Conociendo el tamaño de la muestra de temperaturas, y la probabilidad de éxito, se puede estimar el tiempo de que ocurra el evento de temperaturas [5]-[7], si se encuentran dentro del mismo intervalo, se obtiene con certeza la cantidad de tiempo dentro de una región de aceptación. Esta región de aceptación viene determinada por valores extremos de confianza, que corresponden a valores limites dados por:

$$
t x i, t x s=u t \pm \frac{D t \cdot S D t}{\sqrt{n t}}
$$

Dónde:

txi, txs Tiempo medio requerido $[\mathrm{h}]$.

ut Media muestral del tiempo [h].

nt Cantidad de muestras con respecto al tiempo

SDt Desviación estándar T-Student del tiempo [h].

Dt Distribución T-Student

A partir de la ecuación (7), los tiempos, txi-txt, corresponden al valor inferior y superior, por lo tanto, la diferencia entre estas dos variables se obtiene la durabilidad del tiempo de trabajo muestral:

$$
E(t)=t x i-t x s
$$

Dónde

$\mathrm{E}(\mathrm{t}) \quad$ Durabilidad del tiempo trabajado muestrales [h].

txi Tiempo de nivel de confianza inferior [h].

txs Tiempo de nivel de confianza superior $[\mathrm{h}]$.

\subsection{Formulación del Problema}

Para la formulación del problema se tiene como algoritmo matemático el estudio del consumo de energía eléctrico en sectores comerciales de acuerdo con la arquitectura bioclimática para alcanzar zonas óptimas de confort. La estimación de consumo de energía eléctrica está asociada con la compensación de aire acondicionado y de acuerdo con la arquitectura bioclimática para alcanzar zonas óptimas de confort.

El problema está conformado por dos funciones objetivo, estimar el consumo y satisfacer las necesidades de confort por medio de condiciones óptimas de confort de arquitectura bioclimática y consumo eléctrico en un entorno simulado; además, el problema se encuentra bajo ciertas restricciones: límites de temperatura (exterior e interior), humedad, velocidad del viento, temperatura de sensación térmica, limites arquitectónicos del edificio, limites por temperatura de confort, limites por tiempo de trabajo y ganancias térmicas, todas estas restricciones analizadas por modelo AHSRAE.

Los límites de temperatura (exterior e interior) se consideran entre rango de temperatura mínima y máxima cada 24 horas, los límites de temperatura de confort se encuentran en el rango de $22-25^{\circ} \mathrm{C}$, el rango de humedad se considera entre 40-70\%; los límites de TST están relacionados con los límites de tiempo de trabajo en horas, por último, los limites arquitectónicos del edificio se representan por la ubicación (msnm, latitud, longitud) y caracterices constructivas.

\subsubsection{Cálculo de consumo eléctrico}

Mediante las restricciones del problema y las funciones objetivo, anteriormente descritas en los modelos ASHRAE, Gauss T-Student, el modelo matemático para la evaluación del consumo eléctrico en sectores comerciales de acuerdo a la arquitectura bioclimática para alcanzar zonas óptimas de confort está determinado por:

$$
T S T=\frac{Q T C A}{k A \cdot Q_{\text {caudal }}}+(T K-273.15)
$$

$$
\begin{gathered}
\left.T S T=\frac{\frac{22377406,09 \mathrm{~kJ}}{h}}{\frac{1.012 \mathrm{kj}}{\mathrm{kg}^{\circ} \mathrm{K}} \cdot 3624889,144_{\text {caudal }}}\right) \ldots \\
\ldots+\left(286,71^{\circ} \mathrm{K}-273.15^{\circ} \mathrm{K}\right)
\end{gathered}
$$




$$
\begin{gathered}
T S T=19.67^{\circ} \mathrm{C} \\
k W C a l=\frac{0.2929 \cdot Q T C A}{1000} \\
D T F=\frac{T S T-T r}{T S T-T C} \\
k W P=\frac{k W C a l}{D T} \\
k W P F=\frac{k W P}{(E E R o C O P)} \\
k W P E=\frac{k W P F}{(S E E R o S C O P)}
\end{gathered}
$$

Por ejemplo, tenemos temperatura exterior $16^{\circ} \mathrm{C}, 15$ $\mathrm{km} / \mathrm{h}$ de velocidad de viento y $38 \%$ de humedad a las 17 horas. Se obtiene como resultado $19.67^{\circ} \mathrm{C}$ de TST, aplicando la ecuación (9), obtenidas de ganancias térmicas. A partir del cálculo de TST se puede calcular la cantidad de energía eléctrica se necesita para aumentar o disminuir la temperatura para alcanzar zonas óptimas de confort.

Dónde:

\begin{tabular}{|c|l|}
\hline TST & Temperatura sensación térmica $\left[{ }^{\circ} \mathrm{C}\right]$ \\
\hline QTCA & Ganancia total térmica $\mathrm{J} / \mathrm{h}$ \\
\hline Q_caudal & Cantidad de fluido $\left[\mathrm{m}^{3} / \mathrm{h}\right]$ \\
\hline $\mathrm{kA}$ & Calor especifico del aire $\mathrm{J} / \mathrm{kg}^{\circ} \mathrm{K}$ \\
\hline $\mathrm{TK}$ & Temperatura interna $\left[{ }^{\circ} \mathrm{K}\right]$ \\
\hline $\mathrm{kWCal}$ & Conversión $\mathrm{BTU} / \mathrm{h}$ a $\mathrm{Kw}$ caloríficos. \\
\hline $\mathrm{DTF}$ & Diferencia temperatura acondicionar $\left[{ }^{\circ} \mathrm{C}\right]$. \\
\hline $\mathrm{Tr}$ & Temperatura interior $\left[{ }^{\circ} \mathrm{C}\right]$. \\
\hline $\mathrm{TC}$ & Temperatura Confort $\left[{ }^{\circ} \mathrm{C}\right]$. \\
\hline $\mathrm{kWP}$ & Potencia Calorífica consumida $[\mathrm{kWcal}]$. \\
\hline $\mathrm{kWPF}$ & Potencia Eléctrica consumida nominal $[\mathrm{kW}]$. \\
\hline $\mathrm{kWPE}$ & $\begin{array}{l}\text { Potencia eléctrica consumida por eficiencia } \\
\text { energética }[\mathrm{kW}] .\end{array}$ \\
\hline $\begin{array}{c}\mathrm{EER} \\
\mathrm{COP}\end{array}$ & $\begin{array}{l}\text { Conversión eficiencia de potencia calorífica a } \\
\text { potencia eléctrica. }\end{array}$ \\
\hline $\begin{array}{c}\text { SEER } \\
\text { SCOP }\end{array}$ & $\begin{array}{l}\text { Conversión eficiencia energética aire } \\
\text { acondicionado. }\end{array}$ \\
\hline
\end{tabular}

\subsubsection{Proceso de resolución}

Los resultados, se analiza con el modelo Gauss TStudent, la distribución de TST con respecto a la distribución del tiempo dentro del mismo espacio muestra. Se maneja el software MATLAB, el cual, mediante la incorporación de datos resuelve este modelo de optimización, computando, para el efecto, lo que se conoce como la evaluación del consumo eléctrico en sectores comerciales de acuerdo con la arquitectura bioclimática para alcanzar zonas óptimas de confort.

Tabla 2. Algoritmo para la evaluación del consumo eléctrico en sectores comerciales de acuerdo con la arquitectura bioclimática para alcanzar zonas óptimas de confort.

Paso 1 Adquisición de los datos de temperatura, humedad relativa y velocidad de viento.

Paso 2 Ganancias Térmicas ASHRAE.

\begin{tabular}{ll}
\hline Paso 3 & Determinación de la TST por hora. \\
\hline Paso 4 & Determinación TxS, Txi \\
& Determinación del DTF \\
& Si TST $>25$ \\
& \multicolumn{1}{c}{ DTF $=\frac{T S T-T i n}{T S T-T C}$} \\
Paso 5 & Caso contrario $\quad S i$ TST $<25$ \\
& $\quad$ DTF $=\frac{T C-T r}{T C-T S T}$ \\
& Fin Si \\
\hline Paso 6 & Din Si \\
\hline Paso 7 & Análisis de resultados \\
\hline Paso 8 & Fin \\
\hline \hline
\end{tabular}

La conversión de energía de TST, analiza la ganancia térmica total en unidades de refrigeración $\mathrm{BTU} / \mathrm{H}$, equivalentes a potencia calorífica por la inversa de la eficiencia energética COP, EER. Esto a su vez da al resultado de consumo de energía eléctrica equivalente a la potencia calorífica confort por la inversa de la eficiencia energética SCOP, SEER [41], [42].

Mediante la utilización de MATLAB se pueden estudiar varias opciones de localización del edificio, temperatura, humedad relativa y velocidad del viento, además, otros parámetros arquitectónicos como aislamientos y materiales de construcciones. 


\section{Análisis de Resultados}

La simulación del escenario se conforma por 2 casos de estudio, el primer caso de estudio corresponde desde el 8 de febrero al 7 de marzo del 2018 y el segundo caso desde 8 de agosto hasta 8 de septiembre del 2019.

En las siguientes secciones se detallan las características de los escenarios, las variaciones de temperatura y la evaluación del consumo de energía eléctrica en cada caso de estudio.

\subsection{Característica de los escenarios}

Con el objeto de visualizar las ganancias térmicas del comportamiento bioclimático máximas, obtenidas por el algoritmo ASHRAE [25], [43], [44]. La figura 3, muestra $57 \%$, de color verde por usuarios, de color naranja con $16 \%$, por iluminación, color azul con $13 \%$, conducción de exterior a interior, de color celeste con $9 \%$, por radiación a través de cristales, color amarillo con $4 \%$, equipos eléctricos y menos del $1 \%$ por conducción interna de color azul.

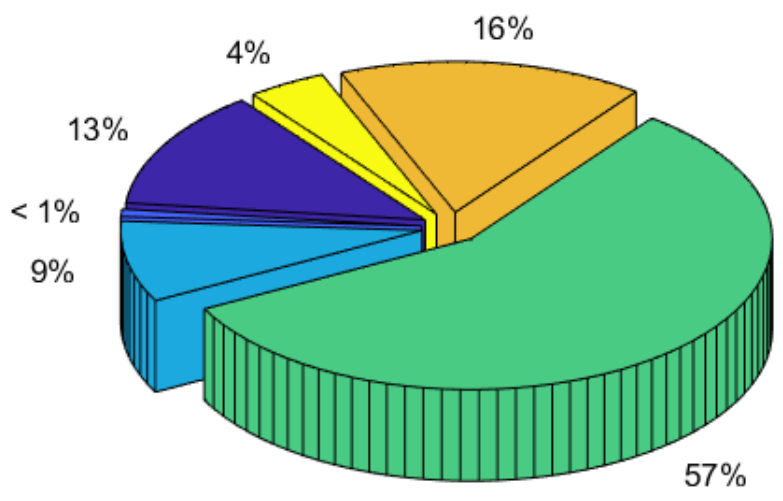

Figura 3. Distribución de cargas térmicas máxima de la Planta.

\subsubsection{Modelamiento Óptimo Gauss, T-Student - Escenario}

El escenario posee temperaturas hasta $31{ }^{\circ} \mathrm{C}$ del exterior, por medio del análisis, los resultados de temperatura de sensación térmica requieren de aire acondicionado, para llegar a una óptima zona de confort. El primer caso de estudio obtiene $19^{\circ} \mathrm{C}$ hasta $34^{\circ} \mathrm{C}$ y el segundo caso alcanza $20^{\circ} \mathrm{C}$ hasta $37^{\circ} \mathrm{C}$ de temperatura de sensación térmica.

La figura 4, representa la distribución de temperaturas del exterior, interior y sensación térmica, con color amarillo, verde y rosa respectivamente de la Planta, que ocurren en 672 horas desde el 8 de febrero al 7 de marzo del 2019. La temperatura de sensación térmica se analiza y corresponde al comportamiento bioclimático y al aprovechamiento ciertas cargas internas y externas.

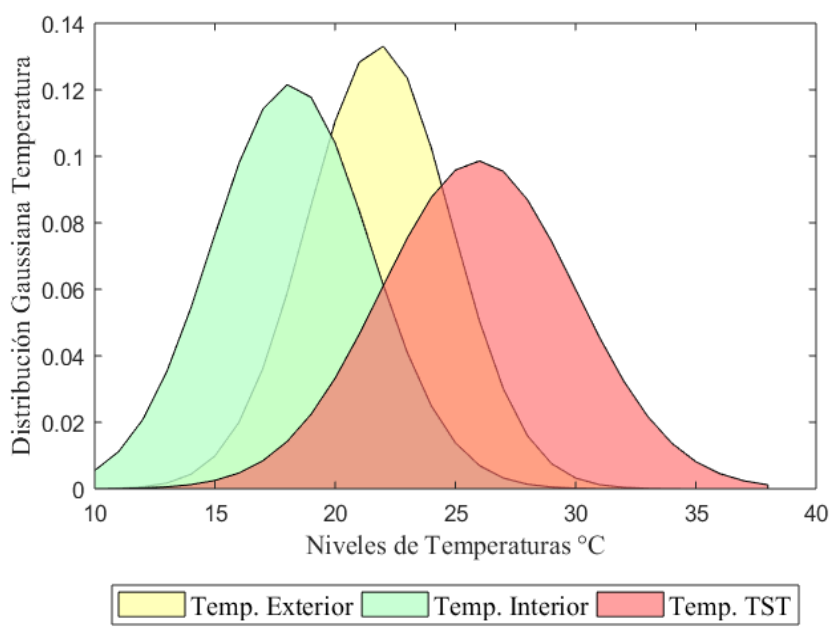

Figura 4. Distribución Gauss de temperaturas primer caso de estudio Guayaquil.

Por lo tanto, el consumo se genera por disminuir la temperatura para alcanzar zonas óptimas de confort. Con el mismo análisis, la figura 5 representa la distribución de las temperaturas del exterior, interior y TST, de la Planta, que ocurren en 768 horas desde el 8 de agosto hasta el 8 de septiembre del 2019.

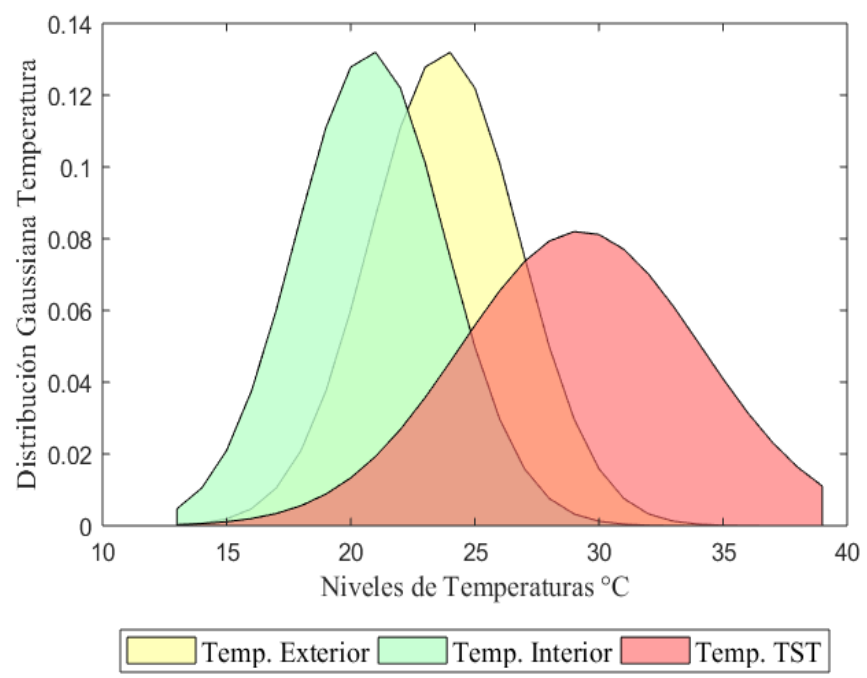

Figura 5. Distribución Gauss de temperaturas segundo caso de estudioGuayaquil.

Las figuras 6 y 7, representan los niveles de temperatura de sensación térmica alcanzadas por 
condiciones bioclimáticas, el aumento de ganancias térmico sirven para determinar la cantidad de energía se requieren para refrigerar y pasar hasta la temperatura de confort [11], [17], [25].

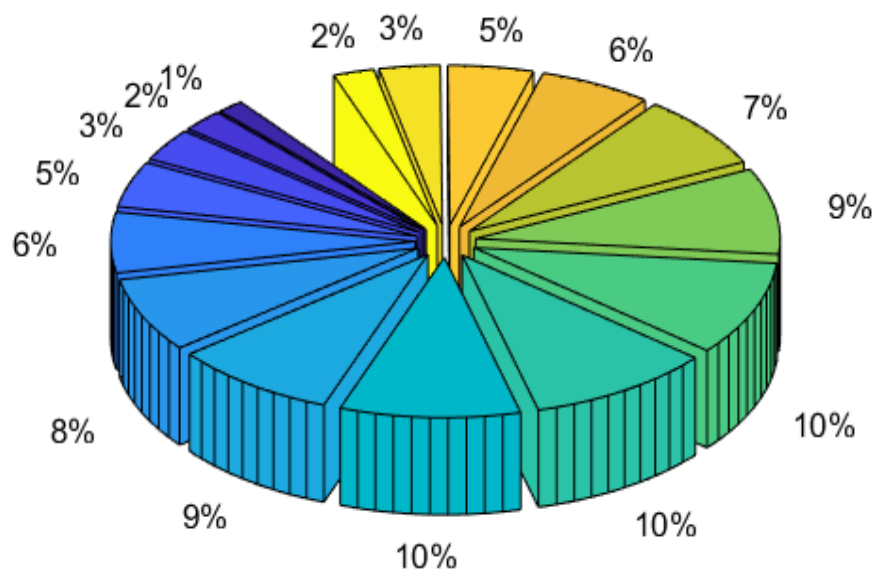

Figura 6. Distribución temperatura TST por hora primer caso de estudio Guayaquil.

En la tabla 3, representa los niveles de temperatura de sensación térmica en el mes de febrero a marzo, se llega a tener $34{ }^{\circ} \mathrm{C}$. La cantidad de horas representa las veces que se repiten las temperaturas dentro del periodo del primer caso de estudio.

Tabla 3. Distribución TST del 8 de febrero al 7 de marzo (2019)-Guayaquil

\begin{tabular}{cccc}
\hline \hline & ${ }^{\circ} \mathrm{C}$ & Horas & $\%$ \\
\hline & 10 & 2 & 1 \\
\hline$\square$ & 11 & 5 & 2 \\
\hline$\square$ & 12 & 25 & 4 \\
\hline$\square$ & 13 & 69 & 6 \\
\hline$\square$ & 14 & 75 & 8 \\
\hline$\square$ & 15 & 62 & 10 \\
\hline$\square$ & 16 & 48 & 11 \\
\hline$\square$ & 17 & 40 & 12 \\
\hline$\square$ & 18 & 29 & 11 \\
\hline$\square$ & 19 & 33 & 10 \\
\hline$\square$ & 20 & 172 & 8 \\
\hline$\square$ & 21 & 81 & 6 \\
\hline$\square$ & 22 & 2 & 4 \\
\hline$\square$ & 23 & 19 & 2 \\
\hline$\square$ & 24 & 10 & 1 \\
\hline
\end{tabular}

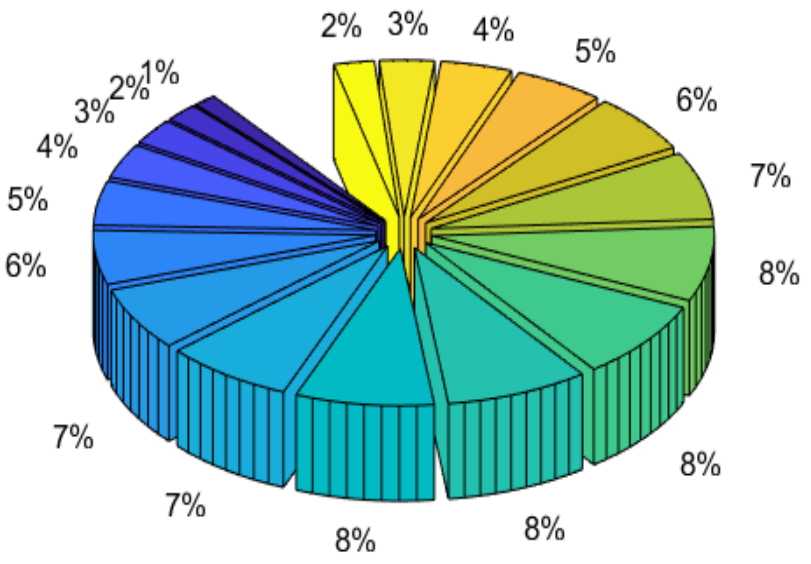

Figura 7. Distribución temperaturas TST segundo caso de estudioGuayaquil.

Tabla 4. Distribución TST del 8 de agosto al 8 de septiembre (2019)Guayaquil

\begin{tabular}{|c|c|c|c|}
\hline & ${ }^{\circ} \mathrm{C}$ & Horas & $\%$ \\
\hline & 10 & 4 & 2 \\
\hline & 11 & 3 & 2 \\
\hline & 12 & 23 & 3 \\
\hline & 13 & 74 & 4 \\
\hline & 14 & 91 & 4 \\
\hline & 15 & 59 & 5 \\
\hline & 16 & 64 & 5 \\
\hline & 17 & 52 & 6 \\
\hline & 18 & 19 & 6 \\
\hline & 19 & 10 & 6 \\
\hline & 20 & 20 & 6 \\
\hline & 21 & 29 & 6 \\
\hline & 22 & 14 & 6 \\
\hline & 23 & 27 & 6 \\
\hline & 24 & 21 & 5 \\
\hline & 25 & 36 & 5 \\
\hline$\square$ & 26 & 28 & 4 \\
\hline & 27 & 39 & 3 \\
\hline & 28 & 39 & 3 \\
\hline$\square$ & 29 & 32 & 2 \\
\hline प & 30 & 36 & 2 \\
\hline$\square$ & 31 & 23 & 1 \\
\hline 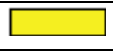 & 32 & 21 & 1 \\
\hline$\square$ & 33 & 4 & $<1$ \\
\hline
\end{tabular}

Mientras en el mes de agosto hasta septiembre como indica la tabla 4 , los niveles de temperatura son muy altos 
llegando alcanzar $37{ }^{\circ} \mathrm{C}$ de sensación térmica, del segundo caso de estudio.

Por lo tanto, para los dos casos de estudio dentro de la Planta es inaceptable laborar en esas condiciones. Estos valores pueden cambiar de acuerdo con el comportamiento de la localidad del edificio, dirección o ubicación de cristales, números de personas laborando internamente, equipos que generen energía térmica.

También los valores de temperatura de sensación térmica pueden variar por las variables climáticas externas por las estaciones del año y del país.

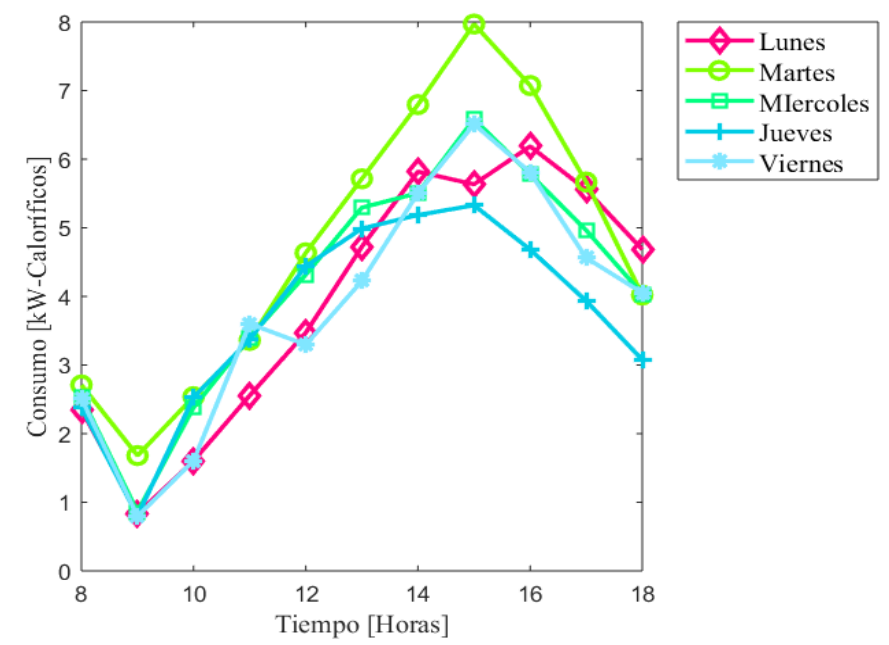

Figura 8. Consumo en kilovatios Caloríficos primer caso de estudio Guayaquil.

La figura 8 y figura 9 , representan el resultado de 5 días, de los dos casos de estudio, como la variación de la energía calorífica para refrigerar de BTU/H a kW Caloríficos por medio de los coeficientes de eficiencia energética [41], [42].

La variación que muestra la figura 8 , obtiene como representación los días: lunes, 0.832 hasta $6.188 \mathrm{kWcal}$; martes, 1.680 hasta $7.969 \mathrm{kWcal}$; miércoles, 0.859 hasta $6.584 \mathrm{kWcal}$; jueves, 0.796 hasta $5.328 \mathrm{kWcal}$; viernes, 0.796 hasta $8.159 \mathrm{kWcal}$.

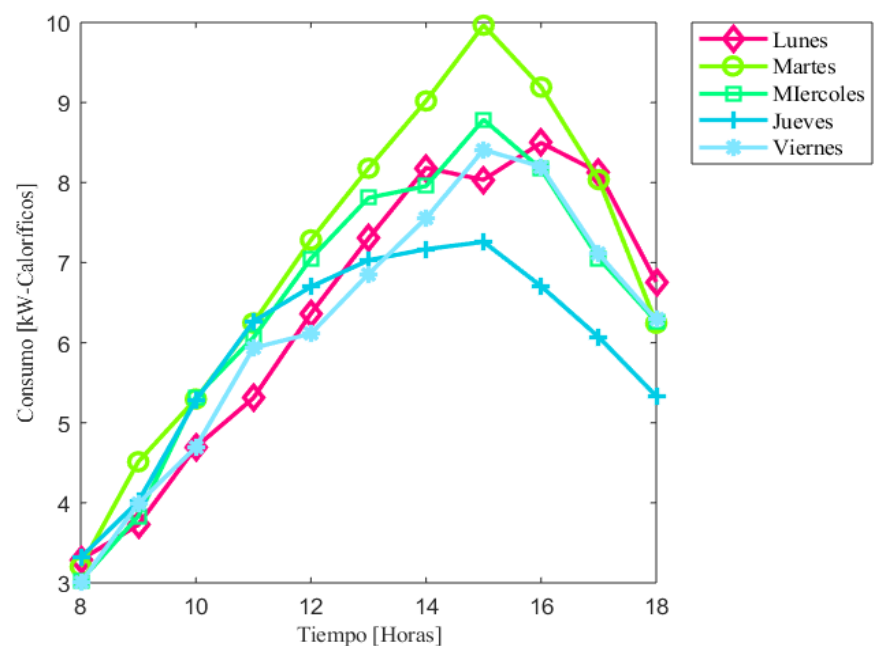

Figura 9. Consumo en kilovatios Caloríficos segundo caso de estudio Guayaquil.

Para el segundo caso, muestra la figura 9 y obtiene como representación los días: lunes, 3.288 hasta 8.497 kWcal; martes, 3.204 hasta $9.966 \mathrm{kWcal}$; miércoles, 3.028 hasta $8.786 \mathrm{kWcal}$; jueves, 3.317 hasta 7.259 $\mathrm{kWcal}$; viernes, 3.001 hasta $8.409 \mathrm{kWcal}$. Los kilovatios caloríficos son la cantidad que necesita eliminar para alcanzar $25 \mathrm{C}^{\circ}$ de confort.

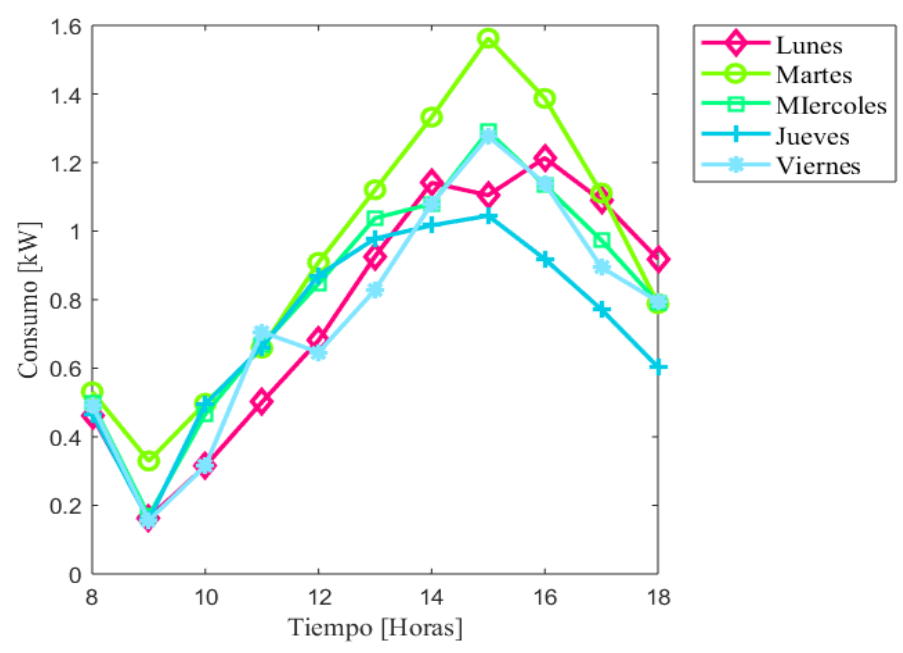

Figura 10. Consumo de Potencia eléctrica kW, alcanzando zonas óptimas de confort primer caso de estudio -Guayaquil.

La figura 10, representa el resultado del consumo de energía eléctrica, obtiene como resultado de los días: lunes, 0.16 hasta $1.21 \mathrm{~kW}$; martes, 0.33 hasta $1.56 \mathrm{~kW}$; miércoles, 0.17 hasta $1.29 \mathrm{~kW}$; jueves, 0.16 hasta $1.04 \mathrm{~kW}$; viernes 0.16 hasta $1.28 \mathrm{~kW}$. 

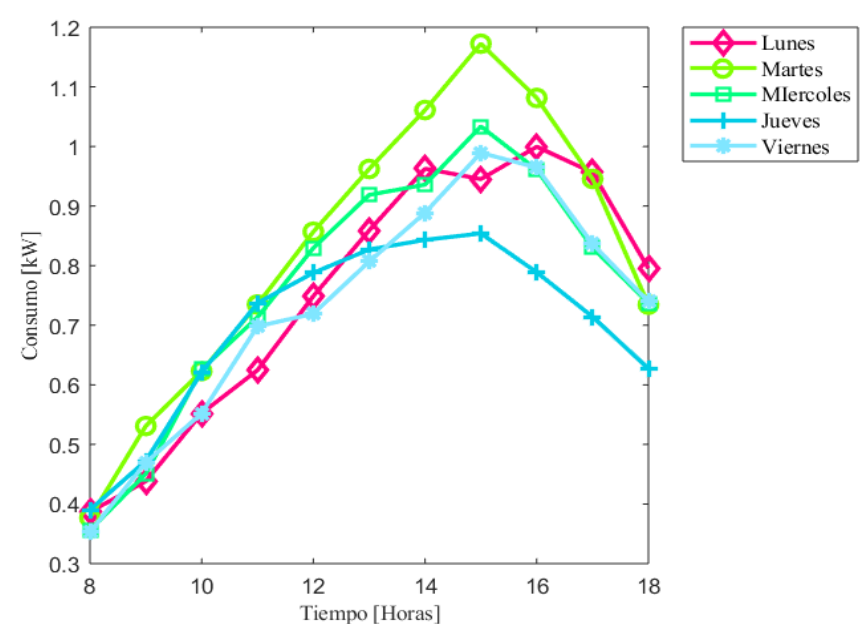

Figura 11. Consumo de energía eléctrica $\mathrm{kW}$, alcanzando zonas óptimas de confort segundo caso de estudio - Guayaquil.

\subsubsection{Consumo total de potencia eléctrica por sistemas óptimos de confort.}

La Tabla 5, son los resultados totales del escenario, de las fechas mencionadas; $X i$, representa el día inicial, $X f$, el día final y $\mathrm{H}$, las horas estimadas de uso de aire acondicionado y $k W H$, el total de energía consumida.

Se debe considerar la capacidad y el número de aire acondicionado a instalar.

En la figura 12, muestra los resultados del segundo escenario. El consumo normal corresponde a la potencia eléctrica consumida por la Planta [23], [24], de color verde. Mientras el consumo agregado por aire acondicionado se representa de color amarillo.

Tabla 5. Consumo total de potencia eléctrica mensual Escenario 2Guayaquil

\begin{tabular}{|c|c|c|c|c|c|c|}
\hline $\mathrm{Xi}$ & Mes & Xf & Mes & Año & $\overline{\mathrm{H}}$ & Kwh \\
\hline 8 & Sep. & 7 & Oct & \multirow{4}{*}{$\underset{\infty}{\stackrel{N}{\infty}}$} & 162 & 326.32 \\
\hline 8 & Oct. & 7 & Nov. & & 162 & 326.32 \\
\hline 8 & Nov. & 7 & Dic. & & 151 & 304.73 \\
\hline 8 & Dic & 7 & Ene. & & 157 & 316.06 \\
\hline 8 & Ene. & 7 & Feb. & \multirow{8}{*}{$\frac{N}{0}$} & 111 & 224.32 \\
\hline 8 & Feb & 7 & Mar. & & 141 & 283.50 \\
\hline 8 & Mar. & 7 & Abr. & & 157 & 316.08 \\
\hline 8 & Abr. & 7 & May. & & 152 & 306.13 \\
\hline 8 & May. & 7 & Jun. & & 159 & 319.33 \\
\hline 8 & Jun. & 7 & Jul. & & 151 & 304.77 \\
\hline 8 & Jul. & 7 & Ago. & & 156 & 314.66 \\
\hline 8 & Ago. & 8 & Sep. & & 161 & 323.74 \\
\hline
\end{tabular}

óptimas de confort segundo escenario.

Como se observa, el consumo de potencia eléctrica para alcanzar zonas óptimas de confort, de acuerdo con la arquitectura bioclimática, por medio de aire acondicionado es el $17 \%$ del consumo normal. El aire acondicionado debe cumplir con estándares de eficiencia energética que disminuya la potencia eléctrica nominal y los costos de utilización [41], [42]. Además, el trabajo estimado toma como diferencia la temperatura de confort y sensación térmica [13].

\section{Conclusiones}

En base a la investigación realizada por medio del modelo Gauss T-Student, se obtuvo el total de 141 horas desde el 8 de febrero hasta el 7 de marzo del 2019 con los cuales se estima el tiempo de trabajo para llegar a $25^{\circ} \mathrm{C}$ de confort con un consumo de energía eléctrica de $283.502 \mathrm{kWh}$. Se obtuvo el total de 161 horas desde el 8 de agosto hasta el 8 de septiembre del 2019, con un consumo de energía eléctrica de $323.746 \mathrm{kWh}$ para alcanzar $25^{\circ} \mathrm{C}$ de confort.

El consumo total de potencia eléctrica para alcanzar zonas óptimas de confort desde el 8 de septiembre del 2018 hasta el 8 de septiembre del 2019 se evalúa por medio del modelo el escenario $13009.732 \mathrm{kWh}, 1494$ horas y del escenario 2, $3666.01 \mathrm{kWh}, 1820$ horas.

Por medio de las condiciones bioclimáticas, se obtienen ganancias térmicas útiles para menorar el 17.7\% del tiempo de uso de aire acondicionado y el consumo de energía eléctrica para alcanzar zonas óptimas de confort.

Mediante el Modelo propuesto, se pueden estudiar varias opciones de acuerdo con localización del edificio, 
propiedades de temperatura, humedad relativa $\mathrm{y}$ velocidad del viento, además, otros parámetros arquitectónicos como aislamientos y materiales de construcciones.

\subsection{Recomendaciones}

Para mejorar el algoritmo se puede recomendar:

Realizar una investigación de otros métodos que puedan brindar soluciones en costos de inversión y costos de consumo de energía eléctrica.

Realizar instalaciones propias de fuentes renovables que satisfagan con la demanda de equipos de aire acondicionado.

Se recomienda instalar aislamientos que permitan almacenamiento de energía interna, y ventilación natural para mejora de arquitectura bioclimática y disminuir el consumo de potencia eléctrica.

\section{REFERENCIAS}

[1] Z. Xu, Q. S. Jia, and X. Guan, "Supply Demand Coordination for Building Energy Saving: Explore the Soft Comfort," IEEE Trans. Autom. Sci. Eng., vol. 12, no. 2, pp. 656-665, 2015.

[2] X. Qin, S. Lysecky, and J. Sprinkle, "A data-driven linear approximation of HVAC utilization for predictive control and optimization," IEEE Trans. Control Syst. Technol., vol. 23, no. 2, pp. 778-786, 2015.

[3] S. Bhattacharya, V. Chandan, V. Arya, and K. Kar, "Demand response for thermal fairness in district heating networks," IEEE Trans. Sustain. Energy, vol. 10, no. 2, pp. 865-875, 2019.

[4] X. Chen, J. Wang, J. Xie, S. Xu, K. Yu, and L. Gan, "Demand response potential evaluation for residential air conditioning loads," IET Gener. Transm. Distrib., vol. 12, no. 19, pp. 4260-4268, 2018.

[5] B. W. Tuinema, M. Gibescu, L. Van Der Sluis, and M. A. M. M. Van Der Meijden, "Probabilistic reliability analysis of future power systems-survey and example," IEEE PES Innov. Smart Grid Technol. Conf. Eur., pp. 1-6, 2011.

[6] F. Coroiu, C. Velicescu, and C. Barbulescu, "Probabilistic and deterministic load flows methods in power systems reliability estimation," EUROCON 2011 - Int. Conf. Comput. as a Tool - Jt. with Conftele 2011, pp. 1-4, 2011.

[7] Y. Huang, Q. Xu, Y. Yang, and X. Jiang, "Numerical method for probabilistic load flow computation with multiple correlated random variables," IET Renew. Power Gener., vol. 12, no. 11, pp. 1295-1303, 2018.

[8] C. J. Wallnerström, J. Setréus, P. Hilber, F. Tong, and L. Bertling, "Model of capacity demand under uncertain weather," 2010 IEEE 11th Int. Conf. Probabilistic Methods Appl. to Power Syst. PMAPS 2010, pp. 314-318, 2010.

[9] B. Jiang, J. Pei, Y. Tao, and X. Lin, "Clustering uncertain data based on probability distribution similarity," IEEE Trans. Knowl. Data Eng., vol. 25, no. 4, pp. 751-763, 2013.

[10] C. F. E. Cancino and A. C. Fuentes, "Hygrothermal comfort within the context of social housing and the perceptions of dwellers [Confort higro-térmico en vivienda social y la percepción del habitante]," Rev. INVI, vol. 30, no. 85, pp. 227-242, 2015.

[11] F. Ferrelli and M. C. Piccolo, "Estudio del confort climático a escala micro-local: El caso de Bahía Blanca (Argentina)," Bitacora Urbano Territ., vol. 27, no. 3, pp. 91-100, 2017.

[12] Ministerio de Desarrollo Urbano y Vivienda del Ecuador, "Eficiencia Energética en Edificaciones Residenciales NEC-HS-EE," p. 40, 2018.

[13] F. B. Silva, A. L. Díaz, and C. J. R. Estébanez, "The use of humidifiers as an energetic alternative to air conditioning: Case of a teaching building in Santiago de Compostela | El uso de humidificadores como alternativa energética al aire acondicionado: Caso de un edificio docente en Santiago de Compost," Rev. Fac. Ing., no. 64, pp. 22-31, 2012.

[14] T. Wang, Y. Xu, C. Withanage, L. Lan, S. D. Ahipasaoglu, and C. A. Courcoubetis, "A Fair and Budget-Balanced Incentive Mechanism for Energy Management in Buildings," IEEE Trans. Smart Grid, vol. 9, no. 4, pp. 31433153, 2018.

[15] R. M. Vignali, F. Borghesan, L. Piroddi, M. Strelec, and M. Prandini, "Energy Management of a Building Cooling System with Thermal Storage: An Approximate Dynamic Programming Solution," IEEE Trans. Autom. Sci. Eng., vol. 14, no. 2, pp. 619-633, 2017.

[16] K. Sano, H. Shimizu, Y. Kondo, and T. Fujimoto, "Improving Accuracy of Temperature Distribution and Energy-Saving Technology of Air Conditioners in Data Centers," IEEE Trans. Components, Packag. Manuf. Technol., vol. 8, no. 5, pp. 811-817, 2018.

[17] R. N. Alejandra and N. Mario, "Análisis del desempeño térmico de los sistemas constructivos de un edificio de oficinas mediante simulaciones dinámicas Thermal analysis of constructive systems at an office building through dynamic simulations," vol. XIX, no. número 3, pp. 279290, 2018.

[18] I. Y. Joo and D. H. Choi, "Distributed Optimization Framework for Energy Management of Multiple Smart Homes with Distributed Energy Resources," IEEE Access, vol. 5, pp. 15551-15560, 2017.

[19] J. A. Pinzon, P. P. Vergara, L. C. P. Da Silva, and M. J. Rider, "Optimal Management of Energy Consumption and Comfort for Smart Buildings Operating in a Microgrid," IEEE Trans. Smart Grid, vol. 10, no. 3, pp. 3236-3247, 2019.

[20] M. Kaddari, M. El Mouden, A. Hajjaji, and A. Semlali, "Reducing energy consumption by energy management and energy audits in the pumping stations," 3rd Renew. Energies, Power Syst. Green Incl. Econ. REPS GIE 2018, 2018.

[21] N. Yaagoubi and H. T. Mouftah, "User-aware game 
theoretic approach for demand management," IEEE Trans. Smart Grid, vol. 6, no. 2, pp. 716-725, 2015.

[22] E. Paniagua, J. Macazana, J. Lopez, and J. Tarrillo, "IoTbased temperature monitoring for buildings thermal comfort analysis," Proc. 2019 IEEE 26th Int. Conf. Electron. Electr. Eng. Comput. INTERCON 2019, pp. 1-4, 2019.

[23] CONELEC, "Regulación modelo de factura," vol. 003, 2012.

[24] A. de R. y control de E. (ARCONEL), "Pliego tarifario para las empresas eléctricas de distribución codificado," Resolución Nro. ARCONEL - 002/19, vol. 18, 2019.

[25] ASHRAE, Handbook of Fundamentals, SI Edition. 2017.

[26] ASHRAE, "ASHRAE Handbook 2001 Fundamentals," Ashrae Stand., vol. 53, no. 9, pp. 1689-1699, 2001.

[27] L. Herrera Sosa, "Temperatura y rangos de confort térmico en viviendas de bajo costo en clima árido seco," Hábitat Sustentable, vol. 3, no. 1, pp. 26-36, 2013.

[28] D. Bravo, F. González, and J. González, "Refrigeración solar de edificaciones. Un estado del arte," Rev. Ing. Constr., vol. 33, no. 2, pp. 115-126, 2018.

[29] G. M. Viegas, P. J. Chevez, G. A. S. Juan, and C. A. Discoli, "Comportamiento energético de mosaicos urbanos representativos (La Plata-Buenos Aires-Argentina): influencia de las variaciones térmicas intraurbanas," Ambient. Construído, vol. 18, no. 3, pp. 175-194, 2018.

[30] J. Ávila-Delgado, M. Robador-González, J. Barrera-Vera, and M. Marrero, ""La influencia del vidrio en el consumo de calefacción, refrigeración e iluminación en rehabilitación de edificios de oficinas bajo clima mediterráneo peninsular (1971-1980) "," Rev. Hábitat Sustentable, vol. 9, no. 1, pp. 68-83, 2019.

[31] A. Kerimray, R. Bektineyev, and L. R. Rojas-Solórzano, "Energy efficiency options for buildings: Insights from buildings energy audit reports in Kazakhstan," IET Conf. Publ., vol. 2016, no. CP688, 2016.

[32] K. Raviraj, N. Gupta, and H. N. Shet, "Analysis of measures to improve energy performance of a commercial building by energy modeling," Proc. 2016 Online Int. Conf. Green Eng. Technol. IC-GET 2016, pp. 1-4, 2017.

[33] H. Sisa and C. Cuji, "Modelo Gaussiano T-Student como método para evaluar el consumo electrico en sectores comerciales de acuerdo a la arquitectura bioclimática para alcanzar zonas optimas de confort," Electr. Eng., vol. 0, no. 0 , p. 0,2020 .
[34] E. H. Piña Hernández, "Prototipo de vivienda vertical social sustentable, enfoque en resistencia al cambio climático," Rev. INVI, vol. 33, no. 92, pp. 213-237, 2018.

[35] I. Ballarini, G. De Luca, A. Paragamyan, A. Pellegrino, and V. Corrado, "Integration of Thermal and Visual Comfort in the Retrofit of Existing Buildings Transformation of an office building into a nZEB," 2018 IEEE Int. Conf. Environ. Electr. Eng. 2018 IEEE Ind. Commer. Power Syst. Eur. (EEEIC / I\&CPS Eur., pp. 1-6, 2018.

[36] A. H. Tan, C. L. Cham, and K. R. Godfrey, "Comparison of Three Modeling Approaches for a Thermodynamic Cooling System with Time-Varying Delay," IEEE Trans. Instrum. Meas., vol. 64, no. 11, pp. 3116-3123, 2015.

[37] B. I. Wang, C. M. Lo, and M. D. Lin, "Building Energy Conservation Strategies Evaluation and Simulation," IEEE Int. Conf. Ind. Eng. Eng. Manag., vol. 2019-Decem, pp. 1874-1878, 2019.

[38] J. Song, G. Xue, Y. Ma, H. A. N. Li, Y. U. Pan, and Z. Hao, "An Indoor Temperature Prediction Framework Based on Hierarchical Attention Gated Recurrent Unit Model for Energy Efficient Buildings," IEEE Access, vol. 7, pp. 157268-157283, 2019.

[39] A. Mosaddegh, C. A. Canizares, and K. Bhattacharya, "Optimal Demand Response for Distribution Feeders with Existing Smart Loads," IEEE Trans. Smart Grid, vol. 9, no. 5, pp. 5291-5300, 2018.

[40] N. Mahdavi, J. H. Braslavsky, M. M. Seron, and S. R. West, "Model Predictive Control of Distributed Air-Conditioning Loads to Compensate Fluctuations in Solar Power," IEEE Trans. Smart Grid, vol. 8, no. 6, pp. 3055-3065, 2017.

[41] INEN, "Eficiencia Energética de acondicionadores de aire2495.pdf." 2012.

[42] MINEN, "Informe Técnico Estándar Mínimo de Eficiencia Energética Equipos de Aire Acondicionado,” pp. 1-74, 2017.

[43] ASHRAE, "Cooling and Heating Load Calculation Manual, 1979.," Cool and Heat Load Calc Man. 1979.

[44] J. Amigo Vásquez, "Ahorro energético en viviendas de países con climas tropicales," Universidad, Cienc. $y$ Tecnol., pp. 1-12, 2006. 